\title{
CO dissociation characteristics on size- distributed rhodium islands on alumina model substrates
}

Cite as: J. Chem. Phys. 108, 2967 (1998); https://doi.org/10.1063/1.475684

Submitted: 03 September 1997 . Accepted: 10 November 1997 . Published Online: 04 June 1998

S. Andersson, M. Frank, A. Sandell, A. Giertz, B. Brena, P. A. Brühwiler, N. Mårtensson, J. Libuda, M. Baümer, and H.-J. Freund

\section{ARTICLES YOU MAY BE INTERESTED IN}

Structure sensitivity in the CO oxidation on rhodium: Effect of adsorbate coverages on oxidation kinetics on $\mathrm{Rh}(100)$ and $\mathrm{Rh}(111)$

The Journal of Chemical Physics 113, 5457 (2000); https://doi.org/10.1063/1.1289764

Atomic and molecular adsorption on Rh(111)

The Journal of Chemical Physics 117, 6737 (2002); https://doi.org/10.1063/1.1507104

Infrared spectroscopic investigation of the rhodium gem-dicarbonyl surface species

The Journal of Chemical Physics 79, 1026 (1983); https://doi.org/10.1063/1.445844

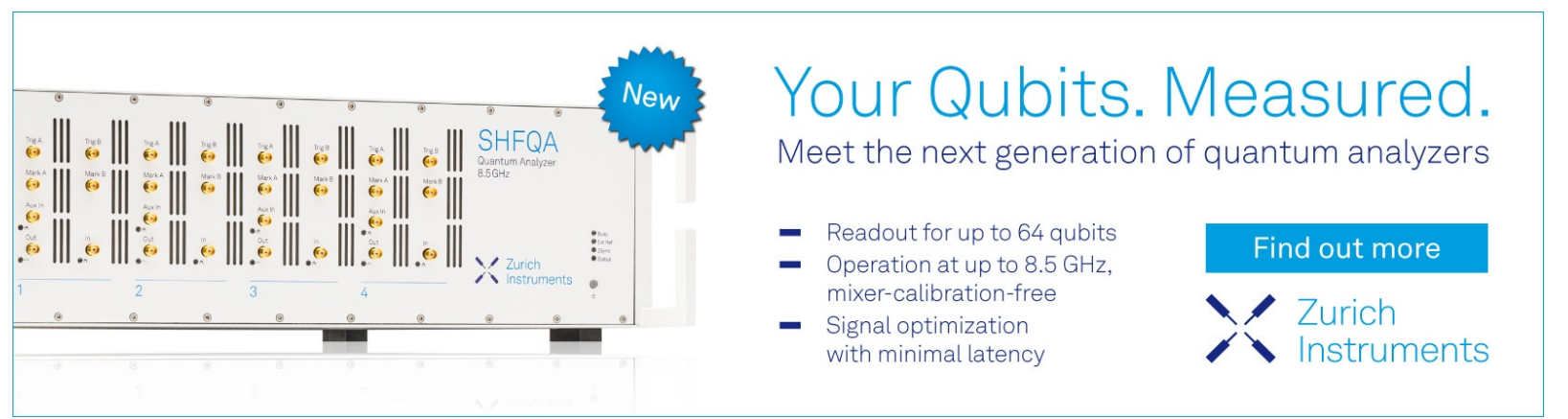




\title{
CO dissociation characteristics on size-distributed rhodium islands on alumina model substrates
}

\author{
S. Andersson \\ Department of Physics, Uppsala University, Box 530, S-751 21 Uppsala, Sweden \\ M. Frank \\ Fritz-Haber-Institut der Max-Planck-Gesellschaft, Faradayweg 4-6, D-14195 Berlin, Germany
}

\author{
A. Sandell \\ Department of Synchrotron Radiation Research, Institute of Physics, Lund University, Box 118, \\ S-221 00 Lund, Sweden \\ A. Giertz, B. Brena, P. A. Brühwiler, and N. Mårtensson \\ Department of Physics, Uppsala University, Box 530, S-751 21 Uppsala, Sweden \\ J. Libuda, M. Baümer, and H.-J. Freund \\ Fritz-Haber-Institut der Max-Planck-Gesellschaft, Faradayweg 4-6, D-14195 Berlin, Germany
}

(Received 3 September 1997; accepted 10 November 1997)

\begin{abstract}
The dissociation of $\mathrm{CO}$ on size-distributed $\mathrm{Rh}$ particles supported on a thin alumina film has been studied with high resolution X-ray Photoelectron Spectroscopy (XPS) and X-ray Absorption Spectroscopy (XAS). Adsorbed CO dissociates upon heating to temperatures above $300 \mathrm{~K}$. The dissociation activity is dependent on the island size, exhibiting a maximum for islands with around 1000 atoms. We have identified size-dependent changes in the $\mathrm{C} 1 \mathrm{~s}$ photoelectron spectra for these $\mathrm{CO}-\mathrm{Rh}$ systems occurring at temperatures lower than the onset of both the dissociation and desorption processes. These changes are interpreted as being due to adsorbed $\mathrm{CO}$ shifting into more highly coordinated sites. The dissociation activity is directly correlated to the availability of these sites, where the observed dissociation is proposed to occur. These results can be interpreted primarily in terms of the size and shape of the deposited Rh particles. (C) 1998 American Institute of Physics. [S0021-9606(98)00607-2]
\end{abstract}

\section{INTRODUCTION}

Metal clusters, both in free form and deposited on surfaces, currently draw increasing attention. A general observation is that fundamental physical properties of the clusters can vary with size. ${ }^{1}$ Of particular interest is the interaction between clusters and molecules, since it has been shown that the efficiency for different catalytic processes may vary with the particle size. ${ }^{2,3}$ As many applied catalysts consist of oxide-supported metal particles, such size-dependent variations could greatly affect the efficiency of the catalytic systems. Thus, detailed knowledge about size-dependent effects could be of great utility for the further development of catalysts.

A fundamental process in heterogeneous catalysis is the dissociation of CO. This step is crucial for, e.g., the FischerTropsch processes (conversion of CO into hydrocarbons). ${ }^{4}$ This has motivated investigations of the fundamental properties of CO dissociation on different substrates using surface science techniques. Many of these studies were initially concentrated on single crystal surfaces of catalytically interesting metals, such as $\mathrm{Pt}$ and $\mathrm{Rh}$. There were discussions on whether CO dissociates on Rh (See, for example, Refs. 5-7 and references therein). It has in any case been shown that $\mathrm{CO}$ dissociates on at least some more open surfaces such as $\mathrm{Rh}(210){ }^{8}$

Due to the technological importance of oxide-supported metal particles, many studies on the interaction between $\mathrm{CO}$ and such systems have also been performed. Previous studies of the $\mathrm{CO}$ dissociation process includes adsorption on supported $\mathrm{Rh}$ particles, and attempts have been made in order to correlate the dissociation activity with particle size. However, Thermal Desorption Spectroscopy (TDS) studies have given seemingly contradictory results. ${ }^{9-11}$

An alternative way to quantify the $\mathrm{CO}$ dissociation process is to directly monitor changes of the adsorbed $\mathrm{CO}$ and the formation of atomic carbon on the surface. This can be done with techniques such as X-ray Photoelectron Spectroscopy (XPS) $)^{8,12}$ and X-ray Absorption Spectroscopy (XAS) utilized in the electron yield mode. The atomic selectivity and the high surface sensitivity of these core level spectroscopies make them very suitable for the study of adsorbates and processes involving these. Industrially produced catalysts are, however, difficult to study with these techniques, due to the amorphous sample structure and because the insulation oxide can cause problems with charging when electron spectroscopic techniques are employed. To avoid these problems, different model systems, well suited for surface science studies, have been developed. For instance, a thin, well-characterized epitaxial $\mathrm{Al}_{2} \mathrm{O}_{3}$ film formed by oxidation of a $\mathrm{NiAl}(110)$ single crystal can be used as a support for the metal particles. ${ }^{13}$ Experiments on metal particles deposited on this type of substrate have 


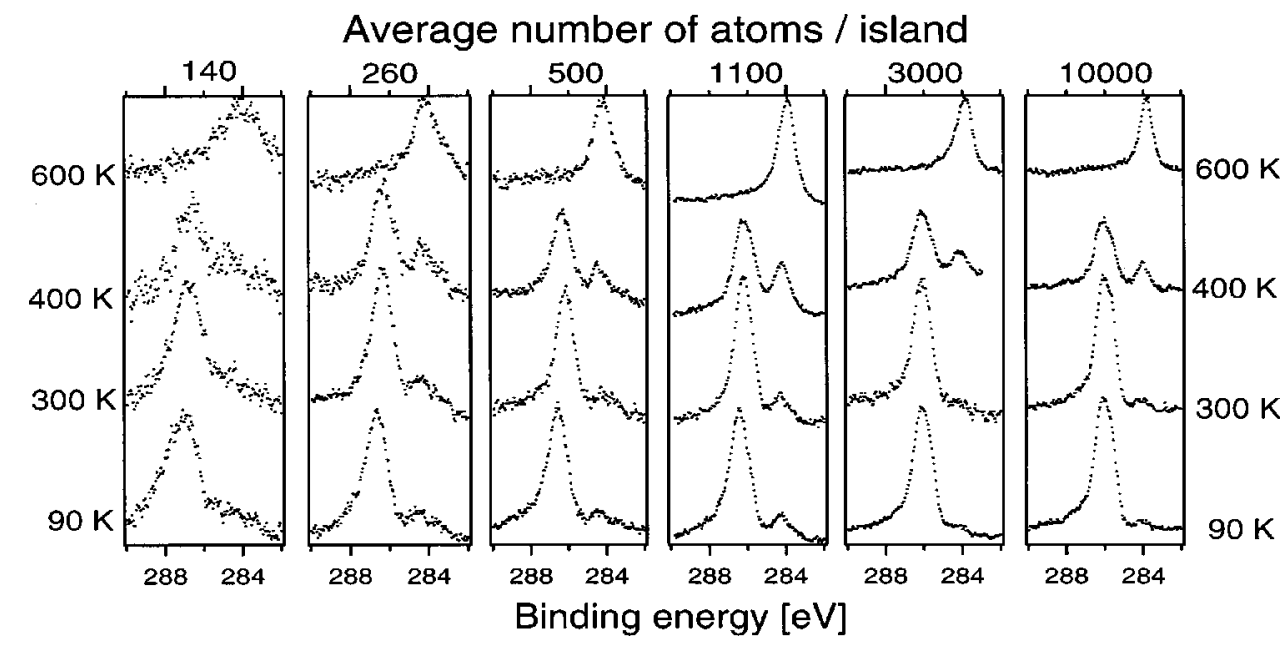

FIG. 1. C $1 s$ photoelectron spectra for $\mathrm{CO}$ adsorbed on islands of the indicated average number of atoms. The spectra are recorded for the original $90 \mathrm{~K}$ adsorbates and after annealing the samples to 300,400 , and $600 \mathrm{~K}$.

shown that it is a suitable support when studying, for example, island growth, ${ }^{14,15}$ size-dependent electronic structure $^{16}$ and reactivity. ${ }^{12,17,18}$

Recently, we presented a study of $\mathrm{CO}$ adsorption on $\mathrm{Rh}$ islands with an average diameter varying from 5 to $30 \AA$ deposited on the thin $\mathrm{Al}_{2} \mathrm{O}_{3}$ film. A fraction of the adsorbed $\mathrm{CO}$ was found to dissociate upon heating, and the dissociation activity was found to increase with increasing island size. ${ }^{12}$ However, a lower activity value reported for single crystal surfaces $\left[10 \%\right.$ dissociation efficiency ${ }^{8}$ for $\left.\mathrm{Rh}(210)\right]$, indicated that there should exist one or more combinations of particle size, surface structure, and, perhaps, support that optimize the probability for dissociation of adsorbed $\mathrm{CO}$.

We have now continued by investigating the $\mathrm{CO}$ dissociation behavior on $\mathrm{Rh}$ particles with sizes covering a much wider range. The results of these studies are presented in two steps: In Ref. 20 we discuss the structural properties of the system and establish that there is a pronounced maximum in the $\mathrm{CO}$ dissociation activity as a function of island size. In this paper we will proceed by elucidating important details of the dissociation process, in particular, the role played by heat-induced changes in the system observed at temperatures lower than the dissociation temperature. We find that two primary types of sites are occupied by the adsorbed $\mathrm{CO}$. We propose that one of the sites is dissociative and that the other is desorptive. We surmise that the proportion of dissociative sites determines the size-dependent dissociation activity in the size range we have studied.

\section{EXPERIMENT}

The experiments were carried out at Beamline 22 at the Swedish synchrotron facility MAX-lab in Lund. The setup at the beamline consists of a modified Zeiss SX-700 monochromator in conjunction with a large hemispherical electron energy analyzer for photoemission and a multichannel plate with a retarding grid for $\mathrm{x}$-ray absorption measurements by the detection of secondary electrons. ${ }^{19} \mathrm{XP}$ spectra were recorded at a photon energy of $380 \mathrm{eV}$ for $\mathrm{C} 1 s$ (total resolution $0.4 \mathrm{eV}$ ). The XPS binding energies are referred to the
Fermi level of the $\mathrm{NiAl}(110)$ substrate. The XA spectra were measured in partial electron yield mode with a photon energy resolution of $0.2 \mathrm{eV}$ at the $\mathrm{C} 1 s$ edge. The absolute photon energies were determined using photoemission spectra excited by first- and second-order radiation. The preparation of the well-ordered $\mathrm{Al}_{2} \mathrm{O}_{3}$ film has been extensively described elsewhere. $^{13}$

Rh (Heraeus, $>99.9 \%$ ) was deposited using a commercial evaporator (Focus EFM 3) based on electron bombardment, which allows evaporation from a rod. During evaporation the sample was put on a retarding potential to avoid effects due to ions produced in the evaporation source that are accelerated toward the sample. The evaporator had previously been calibrated using a quartz microbalance, which gives the nominal film thickness. During deposition the evaporation rate was controlled with the built-in flux monitor. During evaporation the substrate was held at room temperature and chamber pressure was in the range of 5 $\times 10^{-10}-2 \times 10^{-10}$ Torr. Initially the growth of twodimensional $\mathrm{Rh}$ islands is observed, but as the size increases the islands start to grow three-dimensionally. ${ }^{15}$ All the islands studied in this work are expected to have threedimensional character. In the present paper the average number of atoms per island, as estimated through STM measurements, ${ }^{20}$ will be used to denote the different deposits. The average cluster sizes studied in this work range from 140 to more than 100000 atoms per island.

The $\mathrm{Rh}$ islands were subsequently exposed to $40 \mathrm{~L} \mathrm{CO}$ at a temperature of $90 \mathrm{~K}$. This dose should be sufficient to saturate the islands with adsorbed $\mathrm{CO} .{ }^{21}$ The samples were then quickly annealed to different temperatures and thereafter cooled to $90 \mathrm{~K}$ again before the spectra were recorded. The heating-induced changes were studied using XPS and XAS. The temperature of the sample was determined using a K-type thermocouple spot-welded to the crystal, and the heating was performed by passing a current through the tungsten wire holding the sample. 


\section{RESULTS AND DISCUSSION}

\section{A. Size-dependent dissociation activity upon heating}

In this section we use core level photoemission spectra to demonstrate that the $\mathrm{CO}$ dissociation activity on the $\mathrm{Rh}$ particles is size dependent. This is done in a manner similar to that reported earlier, ${ }^{20}$ but we focus on the changes undergone by the adsorbed $\mathrm{CO}$. We will furthermore highlight some features in the spectra that will be of importance for the coming discussion.

Figure 1 shows the evolution of the C $1 s$ XPS peaks for the different rhodium deposits as a function of sample heating. The lowest spectrum in each panel is obtained directly after $\mathrm{CO}$ adsorption at $90 \mathrm{~K}$. The upper three spectra show the effect of annealing to successively higher temperatures, as described in Sec. II. The C $1 s$ spectra are normalized with respect to the background level at a binding energy lower than the $\mathrm{C} 1 s$ peak, so that the intensities after the different heating treatments of each cluster size can be directly compared.

The $90 \mathrm{~K} \mathrm{C} 1 s$ spectra all show a distinct peak at around $286-287 \mathrm{eV}$ due to adsorbed CO. These binding energies are consistent with those found for $\mathrm{CO}$ adsorbed on Rh (111); $286.0 \mathrm{eV}$ for $\mathrm{CO}$ adsorbed on top and 285.4 for $\mathrm{CO}$ in a three-fold hollow site. ${ }^{22}$ In most cases, a small peak at a binding energy of $\sim 284 \mathrm{eV}$ is also present. This peak appears already prior to $\mathrm{CO}$ dosage, and we identify it as atomic carbon. We have no direct explanation to the origin of this peak, but one possibility is that it is caused by dissociation of background $\mathrm{CO}$ during or directly after the $\mathrm{Rh}$ deposition.

The fact that the binding energies shift toward higher values for decreasing island size is a phenomenon generally observed both for clean metal clusters deposited on inert substrates ${ }^{23,24}$ and adsorbates upon these clusters. ${ }^{25}$ The shift can be explained as a combination of several different effects, which are nontrivial to separate. There are initial state effects, such as rehybridizations due to changes in the interaction between the atoms within the cluster and between the cluster and the substrate. There are also final state effects, which in the case of photoemission are very much dependent on the screening properties of the system. In our case, there is a possibility that there is a charge left on the cluster due to inefficient screening from the substrate and there might also occur changes in the ability of the cluster to provide local screening. ${ }^{25,26}$ For the 10000 -atom islands where the shift is expected to be very small the $\mathrm{CO} \mathrm{C} 1 s \mathrm{BE}$ is almost identical to the values found for single crystal substrates.

When the samples are annealed to $300 \mathrm{~K}$ no significant changes in the integrated $\mathrm{CO}$ related $\mathrm{C} 1 s$ intensities are seen. This is in line with previous (TDS) studies, which show that almost no $\mathrm{CO}$ will desorb at temperatures below $300 \mathrm{~K} .{ }^{14}$ However, a shift toward lower binding energy can be observed for the CO peaks. This shift is small for the larger islands, but increases for the smaller islands. The line shape of the peaks also appears to change. These heatinginduced changes will be discussed further in Sec. III B.

After heating to $400 \mathrm{~K}$ an intensity decrease is observed for the $\mathrm{CO}$ related $\mathrm{C} 1 s$ peak. This is caused by desorption

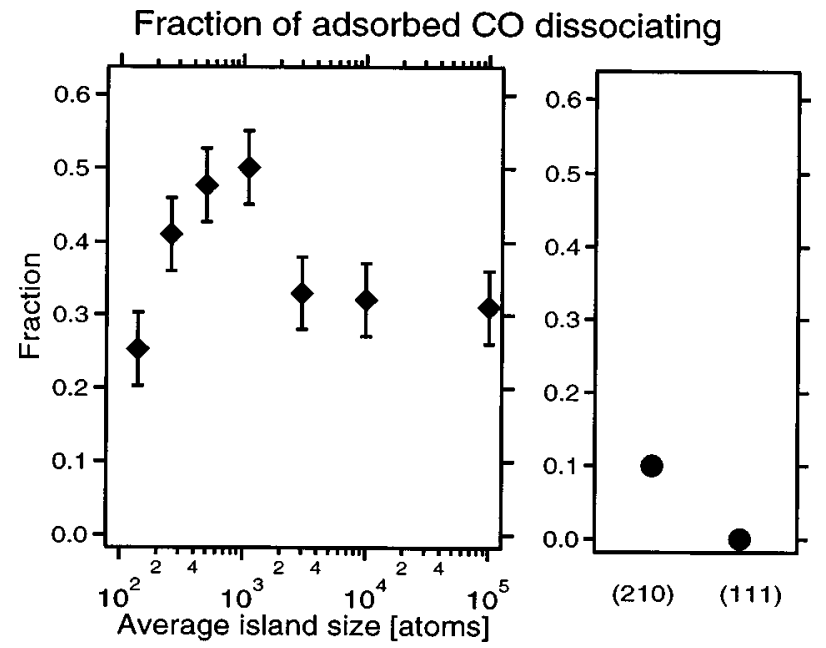

FIG. 2. The $\mathrm{CO}$ dissociation activity, as determined by comparing the original intensities of the $\mathrm{CO}$ peak and the final atomic $\mathrm{C}$ peak (corrected for initial $90 \mathrm{~K} \mathrm{C} 1 s$ intensity) for the different preparations. Efficiency values for $\operatorname{Rh}(210)^{8}$ and $\mathrm{Rh}(111)^{5}$ single crystal surfaces are presented in the graph to the right.

and dissociation of the originally adsorbed CO. The dissociation is evident from the pronounced enlargement of the atomic carbon $\mathrm{C} 1 s$ peak at $\sim 284 \mathrm{eV}$. After annealing to 600 $\mathrm{K}$ all the originally adsorbed $\mathrm{CO}$ has either desorbed or dissociated. In all cases a significant atomic carbon $\mathrm{C} 1 s$ peak remains.

When comparing the areas of the $\mathrm{C} 1 s$ peaks of the originally adsorbed $\mathrm{CO}$ and the final atomic carbon, it can be seen that different amounts of $\mathrm{CO}$ have dissociated in the different cases. To quantify these variations we determine the dissociation fraction as the area of the final atomic carbon peak $(600 \mathrm{~K})$, subtracting any $90 \mathrm{~K}$ atomic carbon contribution, divided by the area of the initial $\mathrm{CO}$ peak $(90 \mathrm{~K})$. The obtained values can be considered to be a measure of the heating-induced dissociation, and are presented in Fig. 2. This is a slightly different quantification of the dissociation than the one used in our other work, ${ }^{20}$ but both methods give the same basic result.

Figure 2 shows that the fraction of $\mathrm{CO}$ that dissociates increases with increasing cluster size up to an average size of 1100 atoms. For islands larger than this the fraction drops again to values around 0.3 , with a slight decrease with increasing size. The tendency for dissociation upon heating thus exhibits a strong size dependence, and a maximum fraction of $\sim 50 \%$ is observed for the 1100 -atom islands.

Single crystal studies have revealed that the fraction of adsorbed CO that dissociates on $\mathrm{Rh}(210)$ is around $0.1,{ }^{8}$ whereas no dissociation is found on the more densely packed $\mathrm{Rh}(100)^{27}$ and $\mathrm{Rh}(111)^{5}$ surfaces. These observations are consistent with the decreasing trend observed in Fig. 2, although the fraction observed for the clusters used in this investigation approaches a value much larger than what has been observed for single crystals. This is probably due to the fact that even the largest clusters in this study still exhibit a large number of steps, kinks, and other defects. ${ }^{15,20}$ 

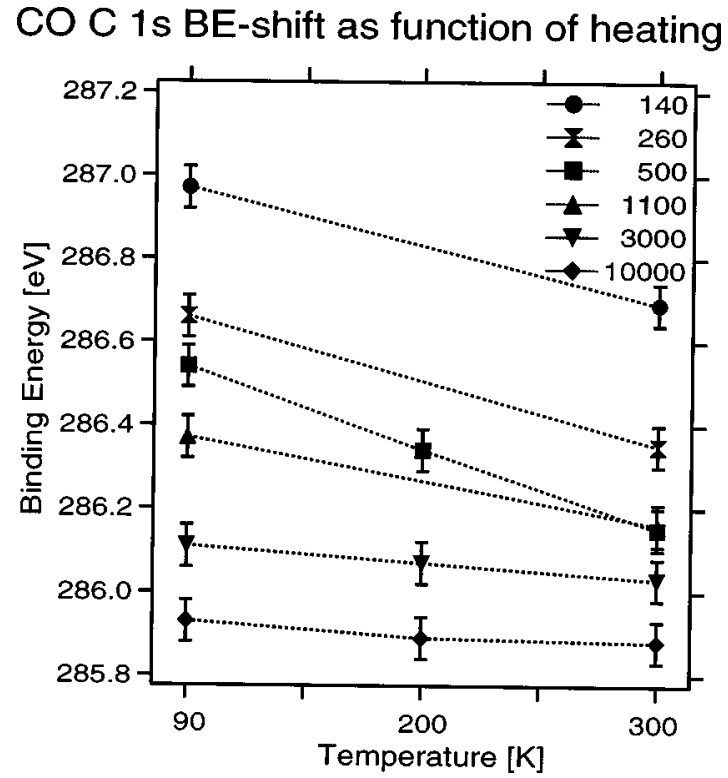

FIG. 3. The temperature-dependent CO C $1 s$ binding energy shifts below the dissociation and desorption thresholds for the different island sizes.

\section{B. Heating-induced changes}

\section{XPS and XAS results}

As mentioned in Sec. III A, heating-induced changes can be seen in the C $1 s$ XP spectra prior to dissociation. Upon heating from 90 to $300 \mathrm{~K}$, the $\mathrm{CO}$ related $\mathrm{C} 1 s$ peak shifts toward lower binding energy, as can be seen by comparing the two lowest spectra for each island size in Fig. 1. To present these results in a more accessible way, Fig. 3 shows the binding energies for the $\mathrm{CO}$ related $\mathrm{C} 1 s$ peaks at 90 and $300 \mathrm{~K}$ for six samples obtained by curve fitting. For three of the samples (500, 3000, and 10000 atoms) spectra were also recorded after heating to an intermediate temperature (200 $\mathrm{K})$. Figure 3 clearly shows that the magnitude of the shift depends on the cluster size: The CO peak shifts very little for the 3000- and 10000-atom islands, whereas there are significant shifts for the smaller clusters. The largest shift, almost $0.4 \mathrm{eV}$, is observed for the 500 -atom islands.

If the line shape of the CO C $1 s$ peak is studied in more detail, a shoulder is seen on the low BE side, indicating that the peak consists of two components. This additional feature was discernible for $\mathrm{CO}$ adsorbed on islands with an average of 500 atoms or more. Measurements with higher resolution, such as the spectrum presented in Fig. 4(a), show this even more clearly. Using curve fitting, ${ }^{28}$ the spectra were decomposed into two different components, denoted A and B, respectively. This was done also for the 140- and 260-atom cases, although the two components could not be discerned in the raw data for these situations.

Figure 4(b) shows the fraction of total CO intensity attributed to component $\mathrm{B}$ for the different sizes at $90 \mathrm{~K}$ and after heating to $300 \mathrm{~K}$. For the $90 \mathrm{~K}$ case, the ratio between the components is similar for all four situations. About $80 \%$ of the CO is associated with "species A" and the other $20 \%$ with "species B." Upon heating, a redistribution of intensity from component A to component B is seen, and this change is noticeably larger for the medium-sized islands. For the
TABLE I. The determined binding energy separation between components $\mathrm{A}$ and $\mathrm{B}$ of the CO C $1 s$ peak.

\begin{tabular}{cc}
\hline \hline Average number of atoms & Observed separation $(\mathrm{eV})$ \\
\hline 140 & $\sim 0.44$ \\
260 & $\sim 0.44$ \\
500 & 0.45 \\
1100 & 0.46 \\
3000 & 0.49 \\
10000 & 0.56 \\
$\mathrm{Rh}(111)$ (Ref. 22) & 0.6 \\
\hline \hline
\end{tabular}

500-atom case the component B has increased to $46 \%$ and for the 1100 -atom case the increase was to $52 \%$ of the total $\mathrm{CO}$ intensity. This clearly shows that the BE shift observed upon heating from 90 to $300 \mathrm{~K}$ is mainly due to a partial transformation of "species A" into "species B." It is furthermore interesting to note that the binding energy separation between the components A and B increases with size, as presented in Table I.

The most remarkable point emerging from this analysis is seen when the $300 \mathrm{~K}$ curve from Fig. 4 is compared with
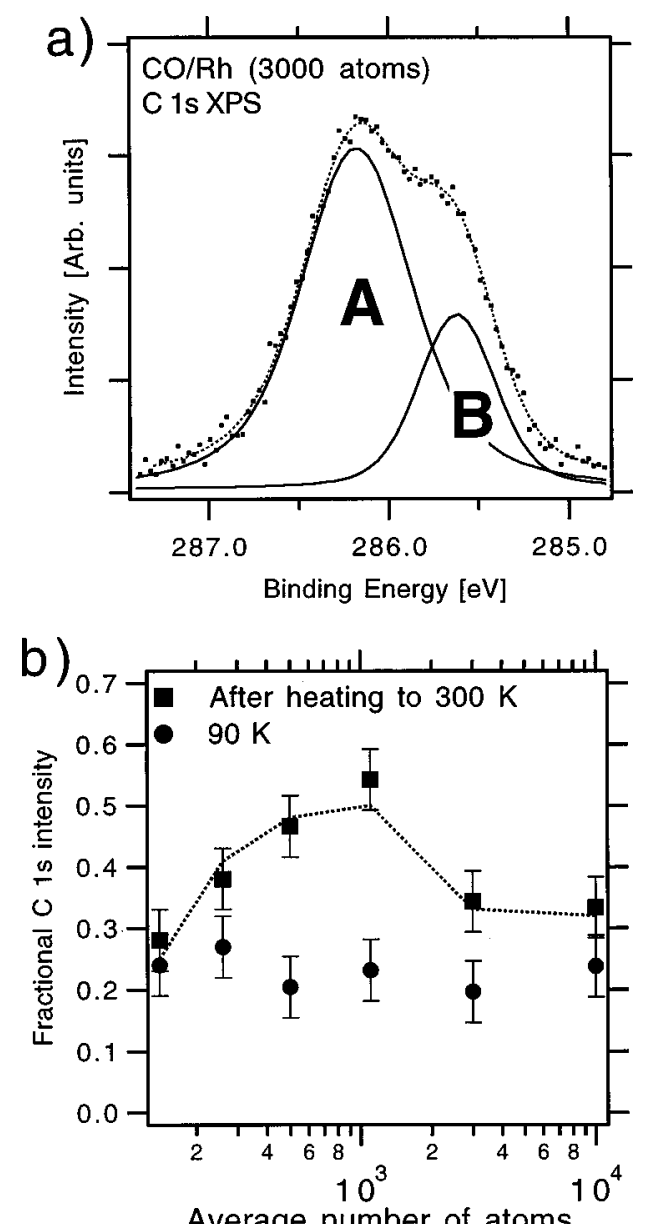

FIG. 4. (a) $\mathrm{C} 1 s$ on 3000 -atom Rh islands after $\mathrm{CO}$ adsorption at $90 \mathrm{~K}$, with a total resolution of $\sim 0.2 \mathrm{eV}$. The areas and shapes of the components $\mathrm{A}$ and $\mathrm{B}$ were determined through curve fitting. ${ }^{28}$ (b) Fraction of $\mathrm{CO}$ in component $\mathrm{B}$ of the $\mathrm{CO}$ peak, at $90 \mathrm{~K}$ and after heating to $300 \mathrm{~K}$. The dotted line shows the fraction of adsorbed $\mathrm{CO}$ ultimately dissociating when the given samples are heated to higher temperatures (see Fig. 2). 


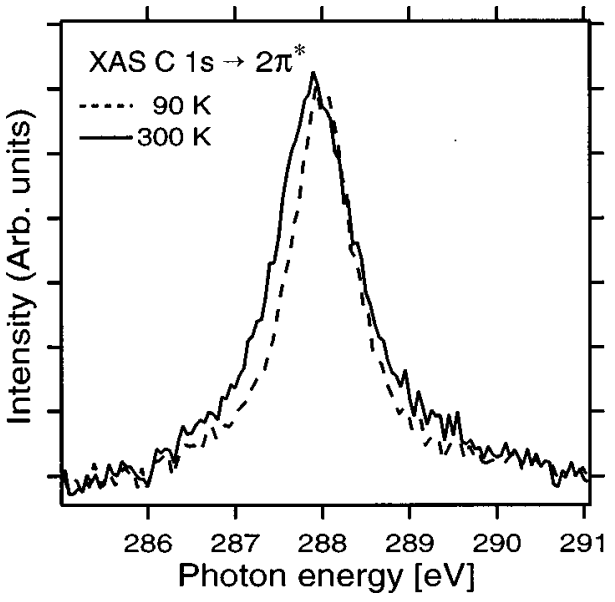

FIG. 5. The XA-resonance peak at $90 \mathrm{~K}$ and after heating to $300 \mathrm{~K}$ for islands with an average of 500 atoms. The intensities were normalized with respect to the resonance peak maximum.

the dissociation curve (Fig. 2), which is included in the figure as a dotted line. The two curves look very similar, indicating that there is a correlation between the occupancy of "species B" and the fraction of CO that dissociates. This correlation will be further discussed in Sec. III C.

Complementary information on the changes observed upon heating from 90 to $300 \mathrm{~K}$ were obtained by X-ray Absorption Spectroscopy (XAS). This technique allows us to probe the initially unoccupied molecular levels of the adsorbed $\mathrm{CO}$ and results in a neutral core-excited state. Figure 5 shows the $\mathrm{C} 1 s \rightarrow 2 \pi^{*} \mathrm{XA}$ resonance peaks at $90 \mathrm{~K}$ and after heating to $300 \mathrm{~K}$ for islands with an average of 500 atoms. After heating, the resonance peak broadens, predominantly toward the low-photon-energy side of the peak. Similar changes were observed for all cases, and in Fig. 6 the widths of the CO C $1 s \rightarrow 2 \pi^{*}$ XA resonances for islands with an average number of atoms from 140 to 3000 are compared. Values are given both for the $90 \mathrm{~K}$ situation and after heating to $300 \mathrm{~K}$.

For the $90 \mathrm{~K}$ case an increase of the $2 \pi^{*}$ resonance width with island size can be seen. Though the shape of the

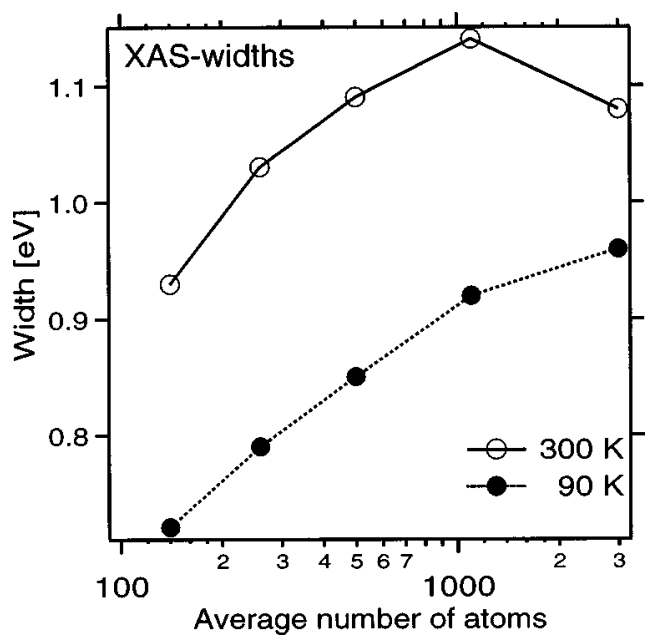

FIG. 6. The widths of the C $1 s \rightarrow 2 \pi^{*}$ XA-resonance peaks (see Fig. 5) as prepared at $90 \mathrm{~K}$ and after flashing to $300 \mathrm{~K}$ as a function of cluster size.
XA spectrum reflects the density of $2 \pi^{*}$ states in the final state, ${ }^{29,30}$ it has been shown that the width of the C $1 s$ $\rightarrow 2 \pi^{*}$ absorption resonance often is correlated to the bonding strength between the $\mathrm{CO}$ and the metal in the ground state, as measured by, for example, TDS. ${ }^{31}$ The observed broadening with increased size could then indicate that the bonding strength between the $\mathrm{CO}$ and the $\mathrm{Rh}$ island increases for larger clusters. However, the size-dependent changes of the resonance widths are rather small compared to what has previously been observed for Pd clusters, ${ }^{25}$ which would indicate that the size-dependent changes in the interaction strength would be larger for Pd. This is also in accordance with TDS data, which shows that CO adsorbed on Pd clusters desorbs at considerably lower temperatures for smaller clusters as compared to large clusters and single crystal surfaces, whereas no such dramatic effect was seen for the Rh islands. ${ }^{14}$

Upon heating to $300 \mathrm{~K}$ the $\mathrm{XA}$ widths increase for all samples, and it is evident that the increase is size dependent. The change in width is largest for $\mathrm{CO}$ on the 1100-atom islands, where the $2 \pi^{*}$ resonance peak is even broader than for the 3000-atom islands. As the broadening occurs mainly on the low-photon-energy side, the peak is also shifted slightly in this direction. This indicates that changes similar to those of the XPS peaks are also undergone by the XAS peak, i.e., intensity is shifted from one component at high photon energy to another, possibly broader, component at lower photon energy. However, due to the lack of features in the $\mathrm{C} 1 s \rightarrow 2 \pi^{*}$ XA-resonance peaks we are unable to conclusively identify any components.

\section{Implications of XPS and XAS results: Predissocialive changes}

Based on the XPS and XAS results, we will now try to explain the existence of the two species and what causes the heating-induced effects seen for heating to $300 \mathrm{~K}$. For this complex system there are a number of properties that may change upon heating, such as the size and geometrical structure of the $\mathrm{Rh}$ islands, the $\mathrm{CO}$ coverage, and the distribution of sites occupied by $\mathrm{CO}$ molecules. However, the island size distribution is expected to exhibit a single maximum, which should not give rise to two discrete C $1 s$ peaks. The shift of the $\mathrm{C} 1 s$ peak to lower BE upon heating could, in principle, be due to coalescence of the islands, but STM shows that this does not occur. ${ }^{20}$ Such a shift would also be expected to be largest for small islands and decrease continuously with increasing size.

Local intra-island restructuring is more difficult to rule out. If we assume that we have the same $\mathrm{CO}$ species, i.e. the $\mathrm{CO}-\mathrm{Rh}$ coordination is unaffected, the screening provided by the $\mathrm{Rh}$ atoms might change upon restructuring by, for instance, a change in the $\mathrm{Rh}$-oxide interaction. However, the XAS data, which essentially monitor the properties of a neutral core-excited $\mathrm{CO}$ species, also show that the $\mathrm{CO}-\mathrm{Rh}$ bonding properties have changed for some of the adsorbed CO. Desorption of some CO could, in principle, contribute to the observed shift, but as the CO C $1 s$ intensity remains constant both desorption and binding energy shifts resulting from it should be negligible. 
CO component intensities as function of treatment

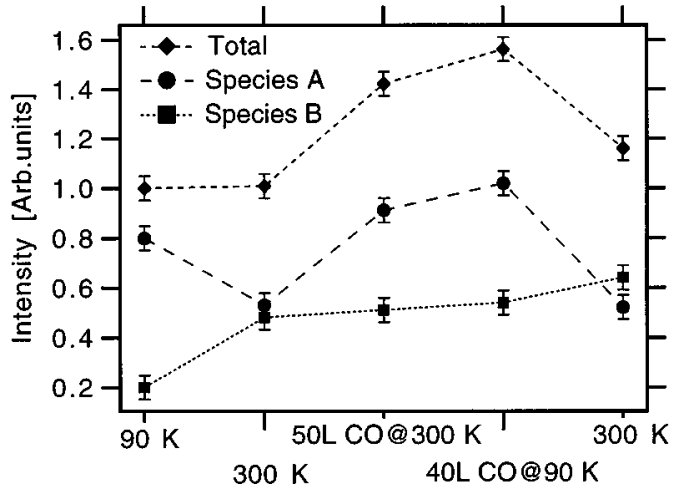

FIG. 7. The CO C $1 s$ intensities for the total CO peak, "species A", and "species B" at different steps in the heating and readsorption process for islands with an average of 500 atoms.

A more reasonable explanation for the appearance of two species is the possibility for $\mathrm{CO}$ to occupy different sites. On $\mathrm{Rh}(111)$, the $\mathrm{C} 1 s$ binding energies are $286.0 \mathrm{eV}$ for $\mathrm{CO}$ in an on-top site and $285.4 \mathrm{eV}$ for $\mathrm{CO}$ in a three-fold hollow site. ${ }^{22}$ This gives a binding energy separation of 0.6 $\mathrm{eV}$, which is close to the separations found here (see Table I. Moreover, temperature-dependent changes have been observed for $\mathrm{CO}$ adsorbed on the $\mathrm{Rh}(111)$ surface, ${ }^{32}$ for which it was shown that heating caused the adsorbed $\mathrm{CO}$ to shift between sites of higher and lower coordination, in a direction dependent on the initial CO coverage. The situation on the (111) surface that most resembles the $90 \mathrm{~K}$ situation on the $\mathrm{Rh}$ islands is found at a $\mathrm{CO}$ coverage of $0.52 \mathrm{ML}$, where $20 \%$ of the CO occupies hollow sites (low-BE component) and the rest are in on-top sites (high-BE component). In this case, it is seen that the population in the low-BE hollow site increases upon heating, but unlike the changes observed on the clusters the changes on the (111) surface are reversible. ${ }^{32}$ It is noteworthy mentioning that NMR studies of $\mathrm{CO}$ adsorbed on Pd clusters have also shown temperaturedependent changes of the adsorption site. ${ }^{33}$

The assignment of the two species as due to $\mathrm{CO}$ in different sites is consistent with the XAS observations. The photon energy positions of the absorption resonance peaks for different species are expected to shift in correlation with the core level XPS peaks. ${ }^{34}$ An increase in the XPS component " $\mathrm{B}$ " at low BE would then be reflected as an increased intensity on the low-photon-energy side. A change to a more highly coordinated site is also expected to cause a broadening of the $\mathrm{CO} 2 \pi^{*}$ resonance peak due to an increased $\mathrm{CO}$ $2 \pi^{*}$-substrate-metal $d$ hybridization. ${ }^{31,34}$ In order to verify that this also applies for $\mathrm{CO} / \mathrm{Rh}$, we measured $\mathrm{C} 1 s \rightarrow 2 \pi^{*}$ XAS spectra for $\mathrm{CO} / \mathrm{Rh}(111)(4 \times 4)$, where $\mathrm{CO}$ only occupies on-top sites ${ }^{22}$ and for $\mathrm{CO}+\mathrm{K} / \mathrm{Rh}(111)$, where $\mathrm{CO}$ only occupies hollow sites. ${ }^{35}$ The XA widths were found to be 1.1 $\mathrm{eV}$ for the on-top site and $1.7 \mathrm{eV}$ for the three-fold hollow sites, supporting the arguments above. It was also found that the XA peak for CO adsorbed in the three-fold hollow site was at a photon energy $\sim 0.35 \mathrm{eV}$ lower than the peak for $\mathrm{CO}$ adsorbed on top.

The characteristic changes in the XPS and XAS spectra

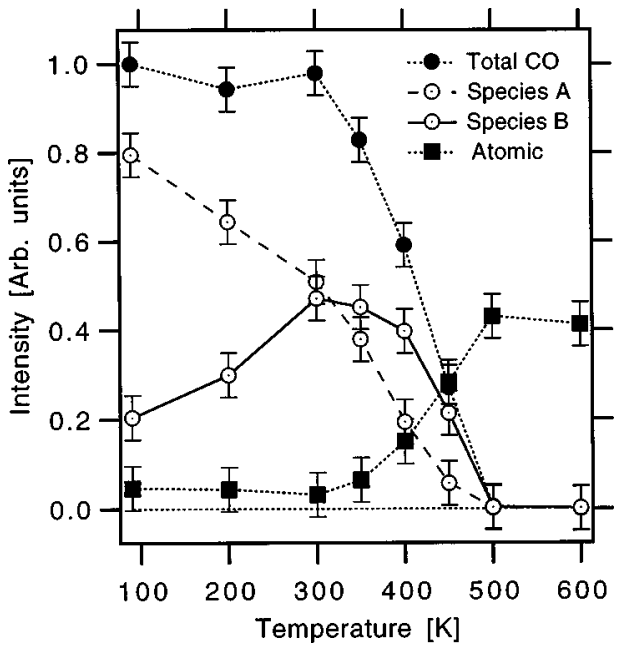

FIG. 8. The determined intensities for the atomic carbon $\mathrm{C} 1 s$ peak, the $\mathrm{CO}$ C $1 s$ peak, and its two components for the different heating steps. The islands consist of an average of 500 atoms.

observed upon heating can thus be interpreted as due to a change of some of the adsorbed $\mathrm{CO}$ from sites with lower coordination ("A") to more highly coordinated sites ("B"). This is confirmed by adsorption of additional $\mathrm{CO}$ after heating to $300 \mathrm{~K}$, as presented in Fig. 7. Upon heating from 90 to $300 \mathrm{~K}$ a decrease of species " $\mathrm{A}$ " and a corresponding increase of species " $\mathrm{B}$ " is observed. When the system is exposed to additional $\mathrm{CO}$, this adsorbs almost exclusively as species " $\mathrm{A}$ ", and the amount adsorbed is comparable to the amount lost due to the transformation upon heating. This can be interpreted as a repopulation of " $A$ " sites left open by $\mathrm{CO}$ moving to " $\mathrm{B}$ " sites. When the system once more is heated to $300 \mathrm{~K}$, most of the additional $\mathrm{CO}$ desorbs, but some is transformed into species " $B$ ". Since the increase of species " $\mathrm{B}$ " is much smaller upon the second heating, we conlude that all available " $\mathrm{B}$ " sites are being occupied.

\section{The dissociation process}

In Sec. III B we have shown that the $\mathrm{CO} \mathrm{C} 1 s$ peak consists of two different components related to $\mathrm{CO}$ adsorbed in two different sites. Upon heating, some of the $\mathrm{CO}$ adsorbed in the less-coordinated sites shifts into more highly coordinated sites. To gain further information about this transformation and about the subsequent heating-induced dissociation process we now take a more detailed look at the intensity changes as a function of temperature. Figure 8 shows the intensities of the two $\mathrm{CO}$ components and the atomic carbon peak as a function of heating for islands with an average of 500 atoms. The total $\mathrm{CO}$ intensity is also included to show changes in the amount of adsorbed $\mathrm{CO}$ in the system. The general trends observed in this figure were also observed for other sizes.

Heating to 200 and $300 \mathrm{~K}$ yields a gradual transformation of species "A" into species " $\mathrm{B}$ ", as discussed in Sec. III B. The total CO intensity remains the same, in agreement with the TDS results. ${ }^{14,36}$ Further heating leads to a decrease in the amount of $\mathrm{CO}$ adsorbed on the islands. This decrease is caused by the competing processes of desorption and dis- 
sociation, as mentioned in Sec. III A. The amount of CO that dissociates is reflected by the increase in intensity of the atomic carbon $\mathrm{C} 1 s$ peak.

At temperatures above $300 \mathrm{~K}$, the $\mathrm{CO}$ intensities of both species " $A$ " and species " $B$ " decrease. The decrease is, however, more rapid for species " $A$ ". If the intensity changes for the two CO components are compared to the intensity changes of the atomic carbon peak, it is seen that the changes for species " $\mathrm{B}$ "' and atomic carbon are inversely correlated. This strongly suggests that it is the $\mathrm{CO}$ adsorbed in sites of type " $\mathrm{B}$ " that dissociates, which is consistent with the trend presented in Sec. III B 1. This conclusion is corroborated by theoretical calculations for $\mathrm{CO}$ dissociation on $\mathrm{Rh}$ surfaces that suggest that $\mathrm{CO}$ is adsorbed in sites with high coordination prior to dissociation. ${ }^{37}$ The intensity decrease of species " $\mathrm{A}$ " is well correlated with the CO desorption, as we show in more detail elsewhere. ${ }^{38}$

Another result supporting the conclusion that it is the $\mathrm{CO}$ adsorbed in sites of type " $\mathrm{B}$ " that dissociates is found if the clusters are exposed to $\mathrm{CO}$ once more after dissociation. The new CO peak consists almost exclusively of species. " $A$ " The most plausible explanation for this is that the sites of species "B" are blocked by the atomic carbon produced by dissociation.

As concluded in Sec. III B, the adsorbed $\mathrm{CO}$ on the $\mathrm{Rh}$ islands consists of two species. We have now shown that upon heating above $300 \mathrm{~K}$, these species exhibit very different behavior. Species "A" is mainly of desorptive character, whereas species " $\mathrm{B}$ " is dissociative.

\section{The dissociative sites}

The previous sections have shown that the sizedependent $\mathrm{CO}$ dissociation activity observed for Rh clusters is correlated to the availability of highly coordinated sites upon the clusters. These sites exhibit a relatively strong metal-CO bond, as was indicated by Fig. 8. However, factors other than the interaction strength between metal and adsorbate are affecting the probability for dissociation of adsorbed $\mathrm{CO}$, as illustrated by the comparison with $\mathrm{CO} /$ $\mathrm{Rh}(111)$. The CO desorption temperature for this surface is similar to those observed for $\mathrm{CO}$ on the clusters. In addition, the XAS resonances for $\mathrm{CO}$ on the (111) surface are broader than for any $\mathrm{CO}$ species on the clusters, which also indicates a stronger CO-Rh bond. ${ }^{31}$ Nevertheless, no dissociation of $\mathrm{CO}$ is seen on the $\mathrm{Rh}(111)$ surface. $^{5}$

Furthermore, the fact that the site changes observed on the clusters are irreversible is a clear indication that the more highly coordinated sites occupied by $\mathrm{CO}$ on the supported clusters are not comparable to the sites available on the (111) surface. This is supported by the smaller BE separation of the two components observed in the $\mathrm{C} 1 s \mathrm{XP}$ spectra for $\mathrm{CO}$ on the $\mathrm{Rh}$ particles, which suggests that the change in coordination between the two different sites is not as large on the islands as on single crystals. The trend of an increased splitting would then be explained by the clusters becoming more regular and ordered with increased size. ${ }^{15,20}$

As previously mentioned, $\mathrm{CO}$ does not dissociate on close-packed crystal surfaces such as $\mathrm{Rh}(111)^{5}$ and $\mathrm{Rh}(100),{ }^{27}$ but does dissociate on at least some more open and stepped surfaces, such as $\operatorname{Rh}(210) .{ }^{8}$ This indicates that steps play an important role in the dissociation process. Based on an atom-probe study of $\mathrm{CO}$ on Rh surfaces, Ren and Liu have suggested that the density of steps is crucial. ${ }^{39,40}$ The Rh clusters in this study are basically threedimensional islands, and, in the initial stages of the growth, the number of steps on the island is expected to increase with island size. Beyond a certain size, however, the islands would be expected to develop more ordered surfaces, and the step density would begin to decrease. Thus, the number of steps on the islands is intuitively expected to change in a way similar to the dissociation activity we observe. It is therefore reasonable to assume that the reactive sites where $\mathrm{CO}$ dissociates are connected to the irregularities on the islands, which was proposed in our previous paper. ${ }^{20}$

In the present study we demonstrate that only a fraction of the CO adsorbs in sites of type "B" at $90 \mathrm{~K}$. Heating then causes more of the adsorbed $\mathrm{CO}$ to populate these sites. As Fig. 4 shows, a larger fraction of the available dissociative sites are initially occupied on both the smallest and the largest clusters in this study. This could be explained by changes in adsorbate mobility with varying island size. On the smaller and larger islands, which have a lower density of steps (and other defects), the adsorbed CO can diffuse to many of the available dissociative sites already at $90 \mathrm{~K}$. For the medium-sized, more irregular, islands, the diffusion barriers for $\mathrm{CO}$ are larger, and a smaller fraction of the available dissociative sites are populated at $90 \mathrm{~K}$. Upon heating to 300 $\mathrm{K}$, the mobility of the adsorbed $\mathrm{CO}$ increases and more of the dissociative sites are populated. Consequently, since more such sites remains available for the clusters in the middle range, the heating-induced changes in the CO-site distribution will become larger for these.

In the size-range studied in this work the varying proportion of dissociative sites, as seen in Fig. 4(b), explains the size-dependent dissociation activity. For clusters smaller than those studied here, other factors would, however, be expected to affect the dissociation activity. As the islands get smaller and more two dimensional, the importance of the $\mathrm{Rh}$-oxide interaction will increase drastically. It has also been shown that a certain minimum $\mathrm{Rh}$ area is required for dissociation to occur, ${ }^{41,37}$ so for very small clusters spatial constraints are expected to affect the dissociation activity. It remains to be seen what role these factors would play for ultrasmall $\mathrm{Rh}$ paricles on the $\mathrm{Al}_{2} \mathrm{O}_{3}$ film.

To summarize this section we have shown that the observed dissociation maximum is caused mainly by the sizedependent variation of the availability of sites favorable for dissociation on the clusters.

\section{CONCLUSIONS}

The heating-induced $\mathrm{CO}$ dissociation activity on $\mathrm{Rh}$ islands supported by a thin $\mathrm{Al}_{2} \mathrm{O}_{3}$ film shows a strong size dependence. The fraction of dissociating $\mathrm{CO}$ increases with size for islands up to $\sim 1000$ atoms, where a maximum is observed. After this point the dissociation probability exhibits a pronounced drop and then continues to decrease slowly with size. 
Size-dependent changes in the $\mathrm{C} 1 s \mathrm{XP}$ spectra for these $\mathrm{CO}-\mathrm{Rh}$ systems, which are strongly correlated with the dissociation efficiency, are observed prior to the onset for dissociation and desorption. These changes can be explained as a shift of adsorbed CO into more highly coordinated sites favorable for dissociation, and these sites are suggested to be connected to irregularities on the Rh surface. We propose that the size dependency of the dissociation activity is mainly caused by the proportion of sites favorable for dissociation on the islands.

\section{ACKNOWLEDGMENTS}

This work has been supported by the Swedish Materials Research Consortium on Clusters and Ultrafine Particles (which is funded by the Swedish National Board for Industrial and Technical Development (NUTEK) and the Swedish National Science Research Council (NFR), Deutsche Forschungsgemeinschaft, Fonds der Chemischen Industrie and Ministerium fur Wissenschaft und Forschung des Landes Nordrhein-Westfalen. The authors thank the staff at MAXlab for invaluable experimental assistance. S. A. would also like to thank Jesper Andersen et al. for access to data prior to publication. M.F. and J.L. thank the Studienstiftung des deutschen Volkes for a fellowship.

${ }^{1}$ W. A. de Heer, Rev. Mod. Phys. 65, 611 (1993).

${ }^{2}$ M. Che and C. O. Bennett, Adv. Catal. 36, 55 (1989).

${ }^{3}$ G. C. Bond, Surf. Sci. 156, 966 (1985)

${ }^{4}$ G. A. Somorjai, Introduction to Surface Chemistry and Calalysis (Wiley, New York, 1994).

${ }^{5}$ J. T. Yates, Jr., E. D. Williams, and W. H. Weinberg, Surf. Sci. 91, 562 (1980).

${ }^{6}$ D. G. Castner, L. H. Dubois, B. A. Sexton, and G. A. Somorjai, Surf. Sci. 103, L134 (1981).

${ }^{7}$ J. T. Yates, Jr., E. D. Williams, and W. H. Weinberg, Surf. Sci. 115, L93 (1980).

${ }^{8}$ M. Rebholz, R. Prins, and N. Kruse, Surf. Sci. Lett. 259, L797 (1991).

${ }^{9}$ V. Matolín, M. H. Elyakhloufi, K. Mašek, and E. Gillet, Catal. Lett. 21, 175 (1993).

${ }^{10}$ V. Matolín, K. Mašek, M. H. Elyakhloufi, and E. Gillet, J. Catal. 143, 492 (1993).

${ }^{11}$ V. Nehasil, I. Stará, and V. Matolín, Surf. Sci. 331-333, 105 (1995).

${ }^{12}$ J. Libuda, M. Frank, A. Sandell S. Andersson, P. A. Brühwiler, M. Bäumer, N. Mårtensson, and H.-J. Freund, in Elementary Processes in Excitations and Reactions on Solid Surfaces, Springer Series in Solid State Sciences (Springer-Verlag, Berlin, 1996), Vol. 121.

${ }^{13}$ R. M. Jaeger, H. Kuhlenbeck, H.-J. Freund, M. Wuttig, W. Hoffman, R. Franchy, and H. Ibach, Surf. Sci. 259, 253 (1991).
${ }^{14}$ J. Libuda, Ph.D. thesis, Ruhr-Universität Bochum, 1996.

${ }^{15}$ M. Bäumer, M. Frank, S. Stempel, J. Libuda, and H.-J. Freund, Surf. Sci. 391, 204 (1997).

${ }^{16}$ A. Sandell, J. Libuda, P. A. Brühwiler, S. Andersson, A. J. Maxwell, M. Bäumer, N. Mårtensson, and H.-J. Freund, J. Electron Spectrosc. Relat. Phenom. 76, 301 (1995).

${ }^{17}$ H.-J. Freund, Angew. Chem. Int. Ed. Engl. 36, 452 (1997).

${ }^{18}$ M. Bäumer, J. Libuda, and H.-J. Freund, in Chemisorption and Reactivity on Supported Clusters and Thin Films, NATO ASI Series E (Kluwer Academic Dordrecht, 1997), Vol. 331.

${ }^{19}$ J. N. Andersen, O. Björneholm, A. Sandell, R. Nyholm, J. Forsell, L. Thånell, A. Nilsson, and N. Mårtensson, Synchr. Rad. News 4, 15 (1991).

${ }^{20}$ M. Frank, S. Andersson, J. Libuda, S. Stempel, A. Sandell, B. Brena, A. Giertz, P. A. Brühwiler, M. Bäumer, N. Mårtensson, and H.-J. Freund, Chem. Phys. Lett. 279, 92 (1997).

${ }^{21}$ This was verified for islands with an average size up to $\sim 1000$ atoms. For these it was found that a dose of $\sim 1 \mathrm{~L} \mathrm{CO}$ at $90 \mathrm{~K}$ was sufficient to achieve the saturation $\mathrm{CO}$ coverage.

${ }^{22}$ A. Beutler, E. Lundgren, R. Nyholm, J. Andersen, B. Setlik, and D. Heskett, Surf. Sci. 371, 381 (1997).

${ }^{23}$ M. G. Mason, Phys. Rev. B 27, 748 (1983).

${ }^{24}$ G. K. Wertheim, S. B. Di Cenzo, and D. N. E. Buchanan, Phys. Rev. B 33, 5384 (1986).

${ }^{25}$ A. Sandell, J. Libuda, P. A. Brühwiler, S. Andersson, A. J. Maxwell, M. Bäumer, N. Mårtensson, and H.-J. Freund, J. Vac. Sci. Technol. A 14, 1546 (1996).

${ }^{26}$ A. Sandell, J. Libuda, P. A. Brühwiler, S. Andersson, M. Bäumer, A. J. Maxwell, N. Mårtensson, and H.-J. Freund, Phys. Rev. B 55, 7233 (1997).

${ }^{27}$ Y. Kim, H. C. Peebles, and J. M. White, Surf. Sci. 114, 363 (1982).

${ }^{28}$ The criterion used for these fits is that there are two components and that their shapes, widths, and separation are the same before and after heating for each separate cluster size.

${ }^{29}$ U. von Barth and G. Grossmann, Phys. Rev. B 25, 5150 (1982).

${ }^{30}$ A. Nilsson and N. Mårtensson, Physica B 208/209, 19 (1995).

${ }^{31}$ O. Björneholm, A. Nilsson, E. O. F. Zdansky, A. Sandell, B. Hernnäs, H. Tillborg, J. N. Andersen, and N. Mårtensson, Phys. Rev. B 46, 10353 (1992).

${ }^{32}$ A. Beutler, E. Lundgren, R. Nyholm, J. N. Andersen, B. Setlik, and D. Heskett, Surf. Sci. (in press).

${ }^{33}$ R. L. Becerra, C. P. Slichter, and J. H. Sinfelt, J. Phys. Chem. 97, 10 (1993).

${ }^{34}$ H. Tillborg, A. Nilsson, N. Mårtensson, and J. N. Andersen, Phys. Rev. B 47, 1699 (1993)

${ }^{35}$ F. Strisland, A. Beutler, A. Jaworowski, R. Nyholm, D. Heskett, B. Setlik, and J. N. Andersen (unpublished).

${ }^{36}$ Above $300 \mathrm{~K}$ an apparent shift in the temperature scale is, however, observed between XPS and TDS data. See Ref. 38 for more details.

${ }^{37}$ A. de Koster and R. A. van Santen, Surf. Sci. 233, 366 (1990).

${ }^{38}$ S. Andersson, M. Frank, A. Sandell, J. Libuda, B. Brena, A. Giertz, P. A. Brühwiler, M. Bäumer, N. Mårtensson, and H.-J. Freund, Vacuum (in press).

${ }^{39}$ D. M. Ren and W. Liu, Surf. Sci. 232, 316 (1990).

${ }^{40}$ W. Liu and D. M. Ren, Surf. Sci. 232, 323 (1990).

${ }^{41}$ W. M. H. Sachtler and M. Ichikawa, J. Phys. Chem. 90, 4752 (1986) 